\title{
Inflammatory bowel disease and superior mesenteric artery thromboembolism
}

\author{
Steven Nicolaides, Abhinav Vasudevan, Daniel Van Langenberg \\ Department of Gastroenterology, Eastern Health, Box Hill Hospital, VIC, Australia
}

While patients with inflammatory bowel disease are known to be at increased risk of venous thromboembolism, the risk of arterial thrombosis is less well recognized. Here, we describe the case of a middle-aged female with a recent diagnosis of Crohn's disease who presented to her local emergency department with acute abdominal pain. Subsequent investigations revealed a thrombus in the superior mesenteric artery resulting in multi-organ infarction requiring major intra-abdominal surgery and extensive resection of segments of small and large bowel. (Intest Res 2020;18:130-133)

Key Words: Inflammatory bowel disease; Ischemia; Thromboembolism

\section{INTRODUCTION}

Inflammatory bowel disease (IBD), particularly CD, is characterized by a state of chronic inflammation that can involve any segment of the GI tract. Furthermore, this inflammatory state can influence multiple organ systems external to the bowel. These include but are not limited to the musculoskeletal, ocular and vascular systems. ${ }^{1}$ One well documented systemic complication is the propensity for the development of venous thromboembolism. ${ }^{2-4}$ Less well documented or understood is the increase in risk of arterial thromboembolism. ${ }^{5}$ Here, we describe a case of a patient, recently diagnosed with CD who subsequently presented with acute severe abdominal pain secondary to a superior mesenteric artery thromboembolism.

\section{CASE REPORT}

A 48-year-old female patient was diagnosed with ileocolonic

Received June 4, 2019. Revised July 4, 2019. Accepted July 28, 2019. Correspondence to Steven Nicolaides, Department of Gastroenterology, Eastern Health, Box Hill Hospital, 8 Arnold Street, Box Hill 3128, Australia Tel: +61-39895-3890, Fax:+61-99822159, E-mail: steven.nicolaides@ easternhealth.org.au
CD after experiencing months of abdominal discomfort, loose stools and bloating. Her initial colonoscopy demonstrated an inflamed intestinal mucosa with a cobblestone appearance and longitudinal ulcerations throughout the colon extending into the terminal ileum with rectal sparing. Based on these findings she was commenced on oral prednisolone. Her symptoms improved initially however upon weaning the prednisolone, she developed recurrence of symptoms. She was subsequently commenced on 6-mercaptopurine $25 \mathrm{mg}$ daily and allopurinol $100 \mathrm{mg}$ daily. Despite therapeutic thiopurine metabolites and 3 months of therapy, she continued to have abdominal pain and loose stools, opening her bowels 5 to 6 times a day, there was no rectal bleeding.

There was no family history of IBD, no prior use of NSAID, oral contraceptive pill or illicit drugs and no recent travel overseas. She was a current smoker with no other risk factors for cardiovascular disease and had a normal BMI. She did not display any extraintestinal manifestations of CD on history or physical examination.

She underwent a second colonoscopy which showed mild patchy colitis throughout the colon with rectal sparing (Fig. 1). Biopsy revealed patchy mild active chronic colitis. There were rare small clusters of histiocytes without well-formed granulo- 
mata (Fig. 2).

Following this, she had an MRI of the small bowel to assess the extent of disease. This demonstrated a $25 \mathrm{~cm}$ segment of distal and terminal ileum with bowel wall thickening and enhancement. There was no proximal dilatation. A decision was made to commence adalimumab.

Two weeks later and just prior to the commencement of anti-TNF therapy, she presented to the local emergency department with severe abdominal pain, vomiting, and watery diarrhea. On examination, she was hemodynamically stable, afebrile and her abdomen was soft with a mildly tender epigastrium but no signs of peritonism. Blood tests revealed anemia with a hemoglobin $113 \mathrm{~g} / \mathrm{L}$, a mild leukocytosis $14.2 \times 10^{9} / \mathrm{L}$, CRP 6 mg/L, ALT 23 IU/L, GGT 31 IU/L, ALP 81 IU/L, albu$\min 36 \mathrm{~g} / \mathrm{L}$, lactate $1 \mathrm{mmol} / \mathrm{L}$, and lipase of $394 \mathrm{IU} / \mathrm{L}$.

An initial CT scan of the abdomen and pelvis did not show any evidence of colitis or bowel obstruction. However, the spleen was heterogenous and showed features consistent with splenic infarction (Fig. 3).

On day 2 of her admission, she developed worsening abdominal pain and rectal bleeding with no relief from opioid analgesia. A thrombophilia and vasculitis screen did not identify a cause and she was treated empirically with therapeutic enoxaparin. By day 4, she had on-going pain refractory to opioid analgesia so a CT angiogram was performed which revealed a nearly occlusive thrombus within the superior mesenteric artery and diffusely thickened loops of small bowel suggestive of ischemia, plus focal areas of nonenhancement within the liver suggestive of hepatic infarction (Fig. 4). There were no features to suggest aortitis.

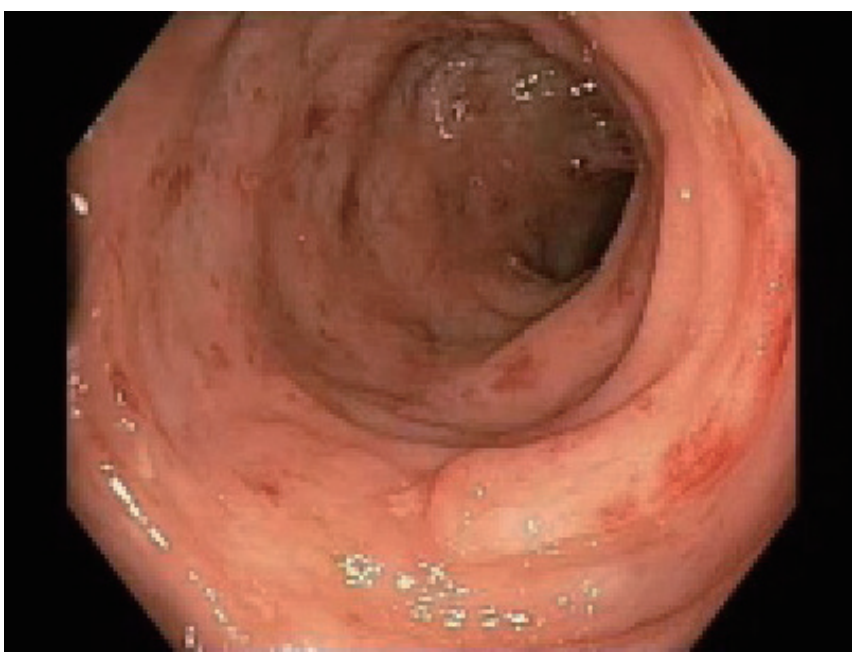

Fig. 1. Colonoscopic finding. Colonoscopy demonstrating patchy colitis throughout the colon.
Lactate was $2 \mathrm{mmol} / \mathrm{L}$ with a $\mathrm{pH}$ of 7.44. White cell count had risen to $18.8 \times 10^{9} / \mathrm{L}$ and hemoglobin had dropped to $95 \mathrm{~g} /$ L, CRP peaked at $13 \mathrm{mg} / \mathrm{L}$, ALT $179 \mathrm{IU} / \mathrm{L}$, GGT $58 \mathrm{IU} / \mathrm{L}$, ALP $88 \mathrm{IU} / \mathrm{L}$ and albumin $16 \mathrm{~g} / \mathrm{L}$. Extensive investigation did not show any evidence of an underlying thrombophilia including protein C or S deficiency, antithrombin III, factor V Leiden, antiphospholipid syndrome and JAK2 gene testing. Serial electrocardiography demonstrated sinus rhythm consistently (no atrial fibrillation or other arrhythmias detected) and transesophageal echocardiogram was unremarkable with no evidence of valvular, atrial or ventricular thrombus and no atrial septal defect or cardiac shunt. There were no clinical features

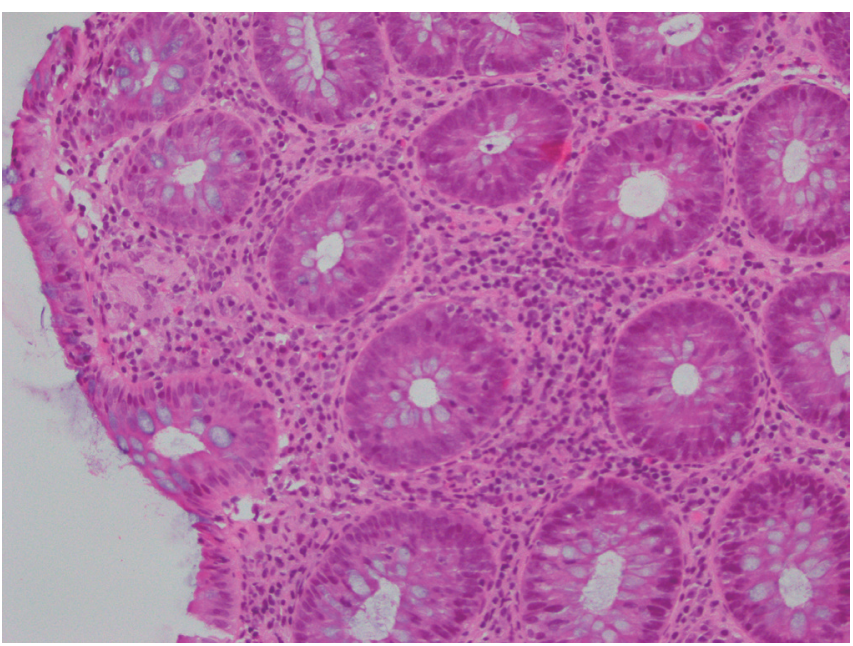

Fig. 2. H\&E stained slides from colonic biopsies $(\times 200)$. The section shows mildly active colitis with increased mixed inflammatory cells in the lamina propria and an occasional cluster of histiocytes beneath the surface epithelium.

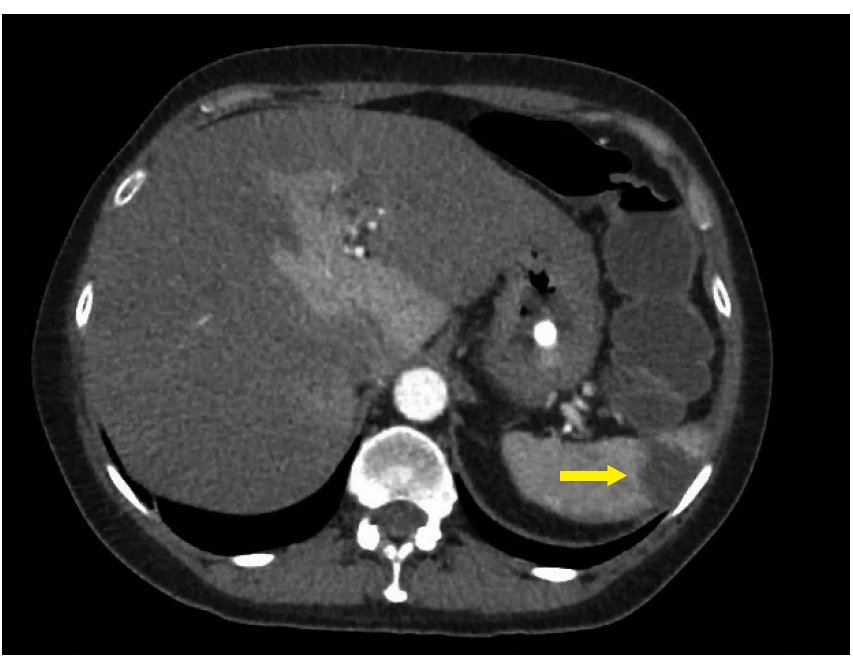

Fig. 3. Abdominal CT finding. CT of the abdomen demonstrates area of infarction within the spleen (arrow). 


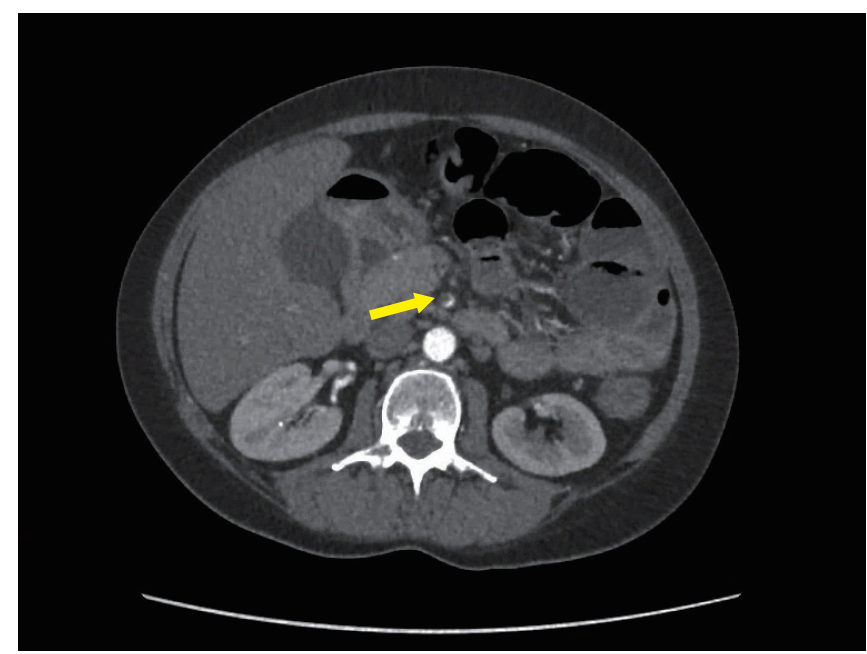

Fig. 4. CT angiogram finding. CT angiogram of the abdomen demonstrates near total occlusion of the superior mesenteric artery (arrow).

of deep venous thrombosis.

She was subsequently commenced on a heparin infusion and underwent a laparotomy, which revealed a dilated, ischemic gallbladder, ischemic caecum, and distal small bowel. An embolectomy of the superior mesenteric artery was performed and a resection of small and large bowel starting 140 $\mathrm{cm}$ from the pylorus to the hepatic flexure leaving $140 \mathrm{~cm}$ of viable small bowel remaining. She was commenced on intravenous antibiotics and 2 days later had a repeat laparotomy showing healthy small and large bowel and a small bowel to transverse colon anastomosis was performed.

Histopathology from the resected specimens was consistent with ischemic colitis (Fig. 5). Her postoperative course was uncomplicated apart from on-going diarrhea likely secondary to short gut syndrome. She required a short period of total parenteral nutrition. Her prednisolone was gradually weaned and long-term warfarin therapy was commenced.

\section{DISCUSSION}

Thromboembolic occlusion of the superior mesenteric artery is recognized to be a common cause of acute mesenteric ischemia. ${ }^{6}$ Despite this there have been few reported cases of superior mesenteric artery thromboembolism related to IBD reported in the literature and most of these relate to chronic ischemia and none of the reported cases resulted in multi-organ infarction. There is one meta-analysis which looked at thromboembolic events and cardiovascular mortality in IBD. ${ }^{2}$ This was a systematic review of observational studies. It found that

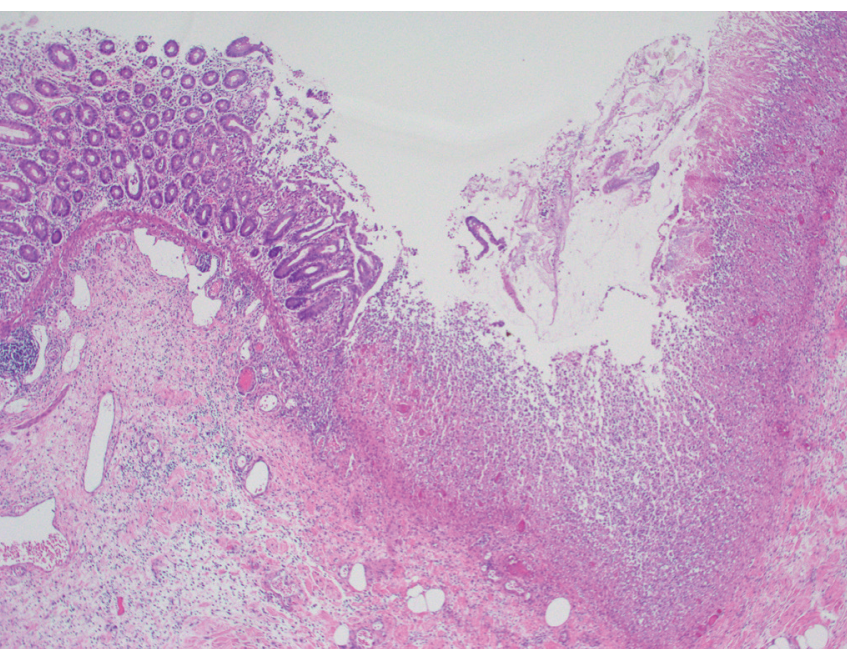

Fig. 5. H\&E stained slides from surgical resection $(\times 40)$. The section shows areas of mucosal necrosis and ulceration with transmural inflammation.

the overall risk of venous thromboembolism in IBD patients was increased almost double that of the general population (RR, 1.96; CI 95\%, 1.67-2.30). ${ }^{7}$ Comparatively a significant increased risk of mesenteric ischemia was observed in IBD patients compared to the general population (RR, 3.46; 95\% CI, 1.78-6.71). ${ }^{7}$ Despite this, there are still conflicting data on the risk of arterial thromboembolism in IBD. While there is an increased risk in other chronic inflammatory diseases such as rheumatoid arthritis, studies have failed to demonstrate any increase in risk of arterial thromboembolism in the IBD cohort. ${ }^{8}$

The causes of acute mesenteric ischemia are usually secondary to an underlying cardiac abnormality either structural or electrophysiological. The other common cause is from any process that increases the general risk of arterial thrombosis including smoking, hypertension, hypercholesterolemia, peripheral vascular atherosclerosis, advanced age or chronic inflammatory disease affecting the vascular system such as vasculitis. $^{9}$

The segment of bowel affected by CD is also particularly susceptible to ischemia given the greater metabolic demand of the inflamed segment. It has previously been noted that mesenteric blood flow is increased in $\mathrm{CD} .^{10}$ Hence, any alteration in the supply of oxygenated blood to affected bowel could result in acute ischemia. The mechanism leading to ischemia may result from either unstable thrombus rupture secondary to increased arterial wall stress or chronic ischemia secondary to the build-up of plaque in those with multiple cardiovascular risk factors. 
This case highlights the difficulty in diagnosis of ischemia in patients with IBD presenting with a suspected disease flare. Delay in diagnosis poses a substantial risk of morbidity and mortality. A high index of suspicion for ischemia is warranted in patients who present with persistent abdominal pain disproportionate to the severity of intestinal inflammation and/ or unresponsive to corticosteroids and analgesia.

\section{FINANCIAL SUPPORT}

The authors received no financial support for the research, authorship, and/or publication of this article.

\section{CONFLICT OF INTEREST}

No potential conflict of interest relevant to this article was reported.

\section{AUTHOR CONTRIBUTION}

Conceptualization: Nicolaides S. Methodology, formal analysis, funding acquisition, project administration, and visualization: Nicolaides S, Vasudevan A, Van Langenberg D. Writing original draft: Nicolaides S. Writing - review and editing: Nicolaides S, Vasudevan A, Van Langenberg D. Approval of final manuscript: all authors.

\section{ORCID}

Nicolaides S https://orcid.org/0000-0003-1726-497X

Vasudevan A https://orcid.org/0000-0001-5026-9014

Van Langenberg D https://orcid.org/0000-0003-3662-6307

\section{ACKNOWLEDGEMENTS}

We thank Dr. Patrick Hosking (Department of Pathology, Eastern Health, Box Hill Hospital, Box Hill, Australia) for providing the report and pictures of the histopathology slides.

\section{REFERENCES}

1. Harbord M, Annese V, Vavricka SR, et al. The first European evidence-based consensus on extra-intestinal manifestations in inflammatory bowel disease. J Crohns Colitis 2016;10:239254.

2. Yuhara H, Steinmaus C, Corley D, et al. Meta-analysis: the risk of venous thromboembolism in patients with inflammatory bowel disease. Aliment Pharmacol Ther 2013;37:953-962.

3. Miehsler W, Reinisch W, Valic E, et al. Is inflammatory bowel disease an independent and disease specific risk factor for thromboembolism? Gut 2004;53:542-548.

4. Kappelman MD, Horvath-Puho E, Sandler RS, et al. Thromboembolic risk among Danish children and adults with inflammatory bowel diseases: a population-based nationwide study. Gut 2011;60:937-943.

5. Novotny DA, Rubin RJ, Slezak FA, Porter JA. Arterial thromboembolic complications of inflammatory bowel disease. Report of three cases. Dis Colon Rectum 1992;35:193-196.

6. Acosta S, Ogren M, Sternby NH, Bergqvist D, Björck M. Clinical implications for the management of acute thromboembolic occlusion of the superior mesenteric artery: autopsy findings in 213 patients. Ann Surg 2005;241:516-522.

7. Fumery M, Xiaocang C, Dauchet L, Gower-Rousseau C, Peyrin-Biroulet L, Colombel JF. Thromboembolic events and cardiovascular mortality in inflammatory bowel diseases: a metaanalysis of observational studies. J Crohns Colitis 2014;8:469479.

8. Aviña-Zubieta JA, Choi HK, Sadatsafavi M, Etminan M, Esdaile JM, Lacaille D. Risk of cardiovascular mortality in patients with rheumatoid arthritis: a meta-analysis of observational studies. Arthritis Rheum 2008;59:1690-1697.

9. McKinsey JF, Gewertz BL. Acute mesenteric ischemia. Surg Clin North Am 1997;77:307-318.

10. Sjekavica I, Barbarić-Babić V, Krznarić Z, Molnar M, CukovićCavka S, Stern-Padovan R. Assessment of Crohn's disease activity by doppler ultrasound of superior mesenteric artery and mural arteries in thickened bowel wall: cross-sectional study. Croat Med J 2007;48:822-830. 